\title{
Structure of the Forward Scattering Amplitude
}

\author{
S. Deser, ${ }^{*}$ Department of Physics, Brandeis University, Waltham, Massachusetts
}

AND

W. Gilbert and E. C. G. Sudarshan, $†$ Lyman Laboratory of Physics, Harvard University, Cambridge, Massachusetts

(Received May 15, 1959)

\begin{abstract}
The matrix elements of various products of two currents between states of equal energy-momentum are studied. Use of the axioms of local field theory leads to an integral representation for the Fourier transform of matrix elements of the retarded commutator in terms of two invariant momentum parameters. Further restriction on the class of allowed functions permits explicit incorporation of the mass restrictions as support conditions on the weight function. The "bound-state" term is separated off and related to the vertex functions. As a simple application, forward-scattering dispersion relations are derived by specialization of the Green's function. In particular, these can be obtained for nucleon-nucleon and $K$-meson-nucleon scattering.
\end{abstract}

\section{INTRODUCTION}

$\mathbf{I}^{\mathrm{N}}$ $\mathrm{N}$ this paper we study the matrix elements of various products of two field operators between two states with the same eigenvalue of the energy-momentum operator. The structure of these matrix elements is analyzed using the general postulates of local field theory: local commutativity, Lorentz invariance, and the existence of a complete set of physical states with positive energies. The asymptotic conditions are implicitly invoked to relate certain matrix elements to the forward scattering amplitude in the physical region. The consequences of these postulates are embodied in an integral representation for the Fourier transforms of these functions in terms of the two invariant momentum parameters. The representation found is not a completely general one, that is, the class of functions so described does not exhaust these postulates. It is, however, significantly wider than that arising in usual perturbation theory.

The basic technique has already been used in a previous paper $^{1}$ (hereafter referred to as I) dealing with the structure of the vertex function. For the Fourier transform of the matrix element of the retarded commutator of two currents we find a representation of the form

$$
\begin{aligned}
\int\left\langle p\left|\left[j_{1}\left(\frac{x}{2}\right), j_{2}\left(-\frac{x}{2}\right)\right]\right| p\right\rangle \theta(x) e^{i k x} d x \\
=\int_{-1}^{1} d \beta \int_{\lambda\left(M_{1} M_{2} \beta\right)}^{\infty} d \mu \frac{H(\mu, \beta)}{k^{2}+2 p k \beta-\mu+i\left(p k+\beta p^{2}\right) \epsilon},
\end{aligned}
$$

where $\lambda\left(M_{1}, M_{2}, \beta\right)$ is a definite function, specified in the text, and $M_{1}, M_{2}$ are the masses of the lowest intermediate states occurring in the decomposition of the matrix element. (We take $\hbar=c=1$ throughout.)

\footnotetext{
* Supported in part by Air Force Office of Scientific Research (ARDC).

$\dagger$ On leave of absence from Tata Institute of Fundamental Research, Bombay, India.

${ }^{1}$ Deser, Gilbert, and Sudarshan, Phys. Rev. 115, 731 (1959).
}

The arbitrariness in the definition of the functional forms in the unphysical region in the $\left(k^{2}, p k\right)$ plane is exploited in writing this representation, since the functions are defined for unphysical as well as physical values of $k^{2}$ and $p k$ by the representation. The weight function $H(\mu, \beta)$ is determined by the values of the Fourier transform of the matrix element in the physical region only. $H(\mu, \beta)$ depends implicitly on $p^{2}$, but this dependence is not analyzed; the information contained in the lower limits $\left(M_{1}, M_{2}\right)$ of the mass spectra of the intermediate states is explicitly incorporated in the representation through the structure of the lower limit of the $\mu$ integration as a function of $\beta$.

We shall find that the integral representation of the time-ordered Green's function everywhere in the physical region can be deduced from that of the commutator matrix element, provided certain "stability" criteria are satisfied. In this case, the commutator matrix element contains all the relevant physical information about the bilinear operator products for the state considered; in the unphysical region of real invariants the representation defines a suitable continuation of these matrix elements. However, if these stability criteria are not satisfied, there is a restricted region of physical values of the $\left(k^{2}, p k\right)$ plane where the values of the time-ordered Green's function cannot be deduced from the commutator matrix element. The stability criteria essentially require that a state whose wave function is the direct product of two wave functions corresponding to the state $|p\rangle$ cannot, through virtual interactions via the field represented by the currents, go into a state of lower rest mass.

It is interesting to notice that these circumstances find a parallel in the propagation characteristics of a wave in a medium (rather than vacuum). If the medium is stable against small perturbations, the "propagation function" which describes the spatiotemporal dependence of a wave propagating inside the medium can be deduced in terms of the "influence function" which describes the wave generated at one point due to the influence of the wave (at a previous 
time) at another point. If, on the other hand, the medium is unstable against small perturbations (of suitable wavelengths and frequencies) its spontaneous emission characteristics constitute additional physical properties of the system, not contained in the influence function. Provided we are considering that part of the propagation where there is no net transfer of energy or momentum between the medium and the wave, the modification of the propagation characteristics due to this critical instability would be confined to a definite region of frequencies and wavelengths. It is precisely this phenomenon that we encounter in the relation between the commutator matrix element ("influence function") and the Green's function ("propagation function").

The specialization of the retarded Green's function to the mass shell of the projectile leads to an integral representation for the forward scattering amplitude. The functions so obtained are not a priori analytic in the upper half-plane of the invariant energy variable; there are possible singularities in the upper half-plane which are, however, confined to the unphysical region. This latter circumstance enables us to define a new function which is analytic in the upper half-plane and which coincides with the physical scattering amplitude in the physical region. It is then trivial to derive dispersion relations for forward scattering. This derivation of forward scattering dispersion relations is applicable in all cases if certain stability criteria are satisfied, including in particular $K$-meson-nucleon scattering and nucleon-nucleon scattering.

It is customary to remark on the relation between the existence of dispersion relations and classical notions of causal propagation. We shall indulge in this only to the extent of pointing out that the usual connections assume that the medium in which propagation is studied is "passive" and does not act as a (fluctuating) source. The on-the-mass-shell stability criteria (which are different from the stability criteria discussed before) are symmetric in the projectile and target masses, and imply that there is no possibility of de-excitation of the "medium" with the emission of a pair of physical "quanta" (i.e., waves satisfying a definite frequencywave number relation). The causal property requisite to the derivation of analyticity for the wave propagation amplitude (sometimes called "the principle of limiting distance") is not satisfied if these stability conditions are violated and we would not expect to find dispersion relations for the wave propagation in such a medium. These qualitative ideas are in fact borne out by the forward scattering amplitude which we deduce by the specialization of our representation.

The domain of validity of the stability criteria is significantly extended by separating, from the integral representations for the operator matrix elements, the contributions from any discrete intermediate states. The mass spectrum of the intermediate states often consists of a discrete line together with a continuum starting at a higher threshold. Methods of separating this contribution are given and the relation between this term and "the coupling constant" term ("renormalized Born approximation") is briefly discussed.

It may be stressed at this point that the problem itself is not new, nor is the idea of constructing suitable integral representations. However, our approach to the problem is somewhat different and the elementary fashion in which mass spectrum restrictions are incorporated is instructive. This result is due in part to the narrower class of functions here considered. Also, we fix the mass of the state $|p\rangle$ at a real positive value throughout the analysis and manipulate the physical function so defined. The dependence on $p^{2}$ is not analyzed further and no analyticity in $p^{2}$ is claimed for either the function represented by the integral or the weight function $H(\mu, \beta)$. In general, the physical mass $\left(p^{2}\right)^{\frac{1}{2}}$ is outside the domain of analyticity, a point which was discussed briefly in I.

At this point, one might consider the extension of this analysis to nondiagonal matrix elements $\left\langle p_{1}\left|\left[j_{1}, j_{2}\right]\right| p_{2}\right\rangle$ and to the dependence of the weight functions on the "external" momenta. However, these investigations are of a somewhat different type and are best treated separately. They form the subject of a subsequent paper, where the generalization of the present techniques is outlined.

Section II deals with the derivation of the integral representation with the restrictions imposed by the lower limits of the mass spectra explicitly incorporated. In Sec. III, the contribution from discrete intermediate states is separated and the connection of this contribution with the vertex function exhibited. The analytic continuation of this contribution, necessary for the existence of dispersion relations, is shown to follow naturally. Dispersion relations for forward scattering are discussed in Sec. IV, while in Sec. V some general comments are made on further properties of the representation.

\section{THE INTEGRAL REPRESENTATION}

Consider a matrix element of the form

$$
C(x)=\left\langle p\left|\left[j_{1}(x / 2), j_{2}(-x / 2)\right]\right| p\right\rangle,
$$

where $j_{1}(x), j_{2}(x)$ are two local "current" operators, which are not necessarily hermitian. From Lorentz invariance it follows that this "diagonal" matrix element is a function of $x$ only through the invariants $x^{2}$ and $p x$, with the property that

$$
C(x)=0 \quad \text { if } \quad x^{2}<0,
$$

since the commutator vanishes for spacelike points. This condition, together with the axiom of completeness of the positive energy states, is sufficient to assure a representation for the Fourier transform of the matrix 
element of the form

$$
\begin{aligned}
f(k)=\int_{-1}^{1} d \beta \int_{-\beta^{2} p^{2}}^{\infty} d \mu \\
\quad \times H(\mu, \beta) \epsilon\left(p k+\beta p^{2}\right) \delta\left(k^{2}+2 p k \beta-\mu\right) .
\end{aligned}
$$

The derivation of this representation is very similar to that presented in I for the vertex function. ${ }^{1}$ The essential point is to notice that the invariant function $f(k)$ depends only on one other (timelike) vector, $p$, and hence admits a Jost-Lehmann representation ${ }^{2}$ of the type

$$
\begin{aligned}
& f(k)=\int_{u^{2}<p^{2}} d^{3} u \int_{0}^{\infty} d t^{2}\left\{\varphi_{1}\left(\mathbf{u}, t^{2}\right)\right. \\
& \left.\quad+\varphi_{2}\left(\mathbf{u}, t^{2}\right) \frac{\partial}{\partial k_{0}}\right\} \Delta_{t}(k-\mathbf{u})
\end{aligned}
$$

with a spherically symmetric weight function in $\mu$ space. We use the freedom, embodied in the Dyson identity, ${ }^{3}$ to convert this representation over a spacelike vector parameter into a representation parametrized with a timelike vector proportional to $p_{\mu}$. The finite limits on the $\beta$ integration in (3) directly reflect the integration over the finite sphere in the $\mu$ space. The representation (3) includes explicitly only causality and the completeness of the positive energy states. The weight function $H(\mu, \beta)$ is not, in general, a Schwartzian distribution; to incorporate the mass restrictions below, we shall confine $H(\mu, \beta)$ to be a tempered distribution. ${ }^{4}$

We now employ the information contained in the mass spectrum to further restrict the range of variation of the parameters $\mu$ and $\beta$. We expand the matrix element (2) over the complete set of eigenstates of energy-momentum vectors.

$$
\begin{aligned}
C(x)=\sum_{n}\left\{\left\langle p\left|j_{1}(x / 2)\right| n\right\rangle\left\langle n\left|j_{2}(-x / 2)\right| p\right\rangle\right. \\
\left.-\left\langle p\left|j_{2}(-x / 2)\right| n\right\rangle\left\langle n\left|j_{1}(x / 2)\right| p\right\rangle\right\} .
\end{aligned}
$$

If $M_{1}, M_{2}$ are the lowest masses that give nonvanishing contributions in each ordering in (5), it follows that for real $k, f(k)$ vanishes unless $(p+k)^{2} \geqslant M_{1}^{2}$ or $(p-k)^{2}$

${ }^{2}$ R. Jost and H. Lehmann, Nuovo cimento 5, 1598 (1957).

3 F. J. Dyson, Phys. Rev. 111, 1717 (1958).

4 This statement is equivalent to the following specialization of the functions used here from those permitted by Jost and Lehmann: In coordinate space, the general functions of the type they consider have the form

$$
\int_{0}^{\infty} d s^{2} \int_{0}^{p^{2}} d u^{2} \frac{\sin \left\{\mu\left[(p x)^{2} / p^{2}-x^{2}\right]^{\frac{1}{2}}\right\}}{\left[(p x)^{2}-p^{2} x^{2}\right]^{\frac{1}{2}}} \Delta_{s^{2}}\left(x^{2}\right) \Phi\left(\mu^{2}, s^{2}\right) .
$$

Use of the Dyson identity brings this into the form

$$
\int_{-1}^{1} d \beta e^{i \beta p x} \int_{0}^{\beta^{2} p^{2}} d u^{2} I_{0}\left\{\left[x^{2}\left(\beta^{2} p^{2}-\mu^{2}\right]^{2}\right\} \int_{0}^{\infty} d s^{2} \Delta_{s^{2}}\left(x^{2}\right) \Phi\left(\mu^{2}, s^{2}\right) .\right.
$$

The class of functions that we are using are those for which the function

$$
g\left(x^{2}\right)=\int_{0}^{\beta^{2} p^{2}} d u^{2} I_{0}\left\{\left[x^{2}\left(\beta^{2} p^{2}-\mu^{2}\right)\right]^{\frac{1}{2}}\right\} \int_{0}^{\infty} d s^{2} \Delta_{8^{2}}\left(x^{2}\right) \Phi\left(\mu^{2}, s^{2}\right)
$$

is expandable in the complete set $\Delta_{r^{2}}\left(x^{2}\right)$, i.e., those functions $g\left(x^{2}\right)$ which are sufficiently well-behaved for large values of $x^{2}$.

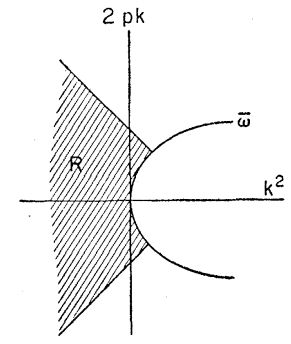

Case A

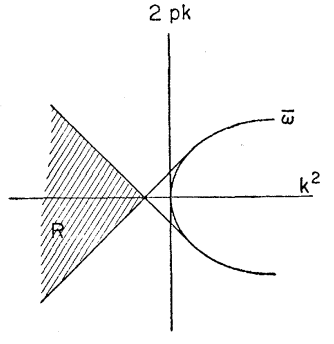

Case B
FIG. 1. The region $\Omega$, in which the Fourier transform vanishes, in the $\left(k^{2}, 2 p k\right)$ plane.

$\geqslant M_{2}^{2}$. For all real vectors $k,(p k)^{2} \geqslant p^{2} k^{2}$, and so all physical values of the invariants lie outside the parabola $\bar{\omega}$ in the $\left(k^{2}, 2 p k\right)$ plane. The mass conditions are that $f\left(k^{2}, p k\right)$ vanishes in the region $R$, that part of the $\left(k^{2} p k\right)$ plane outside $\bar{\omega}$ where $k^{2}+2 p k \leqslant M_{1}^{2}-p^{2}$; $k^{2}-2 p k \leqslant M_{2}^{2}-p^{2}$.

Two distinct cases arise (see Fig. 1) : Case $A: M_{1}+M_{2}$ $\geqslant 2 \kappa$, the two physical regions in which $f\left(k^{2}, p k\right)$ does not vanish are disjoint and the region $Q$ has part of the parabola $\bar{\omega}$ as its boundary. Case $B: M_{1}+M_{2} \leqslant 2 \kappa$ and the physical regions overlap.

Case $A$ is the natural one and holds if, in particular, the particle states are stable in a sense that we will discuss later.

For every point in the $\left(k^{2}, p k\right)$ plane the function $f\left(k^{2}, p k\right)$ receives contributions from the integral (3) along a line in the $(\mu, \beta)$ plane given by the equation

$$
k^{2}+2 p k \beta-\mu=0 \text {. }
$$

The parabola $\bar{\omega}$ in the $\left(k^{2}, p k\right)$ plane generates a set of lines in the $(\mu, \beta)$ plane which have as their envelope the parabola $\mu=-\beta^{2} p^{2}$. The part of the line $k^{2}+2 p k$ $=m_{1}^{2}-p^{2}$ in the physical region generates a pencil of lines through $\left(p^{2}-m_{1}^{2},-1\right)$ lying between the tangent of positive slope and the line $\beta=+1$. All other points

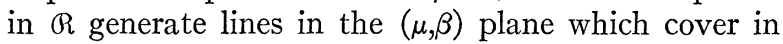
the strip $-1 \leqslant \beta \leqslant 1, \mu \geqslant-\beta^{2} p^{2}$ only the region already swept out by these two pencils. The set of points along a line $p k=b$ generates a set of parallel lines. All the lines generated by points in $R$ intersect the parabola $\mu=-\beta^{2} p^{2}$ (or at least touch it, in Case $A$ ) and the $\epsilon$-function in (3) changes sign inside the parabola (or at the point of contact) where $H(\mu, \beta)$ vanishes.

These properties are sufficient to enable us to use a lemma of Jost and Lehmann ${ }^{2}$ to conclude that the representation is unique and that the support of the weight function $H(\mu, \beta)$ is in the region $\delta$ consisting of the part of the strip $\mathcal{S}^{\prime}: \mu \geqslant-\beta^{2} p^{2} ; 1 \geqslant \beta \geqslant-1$ not covered by the lines generated by points in $R$. The regions for the two cases are

$$
\begin{aligned}
& \mu \geqslant \lambda\left(M_{1}, M_{2}, \beta\right)=\max \{\left(M_{1}-\kappa\right)^{2}+2 \kappa\left(M_{1}-\kappa\right) \beta, \\
&\left.\left(M_{2}-\kappa\right)^{2}-2 \kappa\left(M_{2}-\kappa\right) \beta\right\}, \\
& 1 \geqslant \beta \geqslant-1 \quad \text { if } 2 \kappa<M_{1}+M_{2},
\end{aligned}
$$




$$
\begin{aligned}
& \mu \geqslant \lambda\left(M_{1}, M_{2}, \beta\right)=\max \left\{\frac{1}{2}\left(M_{1}^{2}+M_{2}^{2}\right)-\kappa^{2}\right. \\
& \left.+\frac{1}{2}\left(M_{1}^{2}-M_{2}^{2}\right) \beta,-\beta^{2} \kappa^{2}\right\}, \\
& 1 \geqslant \beta \geqslant-1 \text { if } 2 \kappa>M_{1}+M_{2},
\end{aligned}
$$

and are illustrated in Fig. 2.

It is to be emphasized at this stage that, to start with, we know the Fourier transform of the function $C(x)$ only for real values of $k$ and hence the function $f\left(k^{2}, p k\right)$ only for the arguments outside the parabola $(p k)^{2}=k^{2} p^{2}$. The representation (1), however, uniquely defines an extension of this function for all real values of the invariants. In particular, for Case $A$, the function so defined vanishes in the unphysical segment sliced off by the chord joining the points of intersection of the two boundary lines with the parabolic boundary.

On the other hand, for extreme values of the masses the contribution to the matrix element from a class of intermediate states has to vanish in the physical region: For example if $M_{1}+\kappa<M_{2}-\kappa$, the contributions of the class $\left\langle p\left|j_{1}(x / 2)\right| n\right\rangle\left\langle n\left|j_{2}(-x / 2)\right| p\right\rangle$ vanish for all states which satisfy $\left(M_{1}+\kappa\right)^{2}<n^{2}<\left(M_{2}-\kappa\right)^{2}$.

At this juncture we should remark that our representation for the commutator matrix element as well as for the Green's function is incomplete since sufficient attention has not been paid to the (improper) limit of these expressions as $x \rightarrow 0$. These singularities are of the nature of $\delta$-functions or their derivatives of finite order and correspond in general to structures for the commutator of the form:

$$
\begin{aligned}
\int d \beta d \mu \epsilon\left(p k+\beta p^{2}\right) \delta\left(k^{2}+\right. & 2 p k \beta-\mu) \\
& \times \sum_{\lambda=1}^{N} H_{n}(\mu, \beta)\left(p k+\beta p^{2}\right)^{n},
\end{aligned}
$$

where the summation is finite.

That the weight function $H(\mu, \beta)$ in our representation is real, irrespective of the nature of the currents, can be shown to follow from $T P$, or equivalently $C$, invariance. From the representation for the matrix element of the commutator, one may immediately write down the representation for the corresponding matrix element of the retarded commutator in the form:

$$
\begin{array}{r}
G_{\mathrm{ret}}(k)=i \int d x \theta(x) e^{i k x}\left\langle p\left|\left[j_{1}(x / 2), j_{2}(-x / 2)\right]\right| p\right\rangle \\
=\int_{\mathcal{S}} d \beta d \mu \frac{H(\mu, \beta)}{k^{2}+2 p k \beta-\mu+i\left(p k+\beta p^{2}\right) \epsilon} .
\end{array}
$$

On the other hand, the time-ordered Green's function,

$$
G_{+}(k)=i \int d x e^{i k x}\left\langle p\left|\left(j_{1}(x / 2) j_{2}(-x / 2)\right)_{+}\right| p\right\rangle
$$

is not so simply ${ }^{2}$ related to the expression for the commutator. Nevertheless in the normal case $A$, it is indeed possible to write down a representation for this
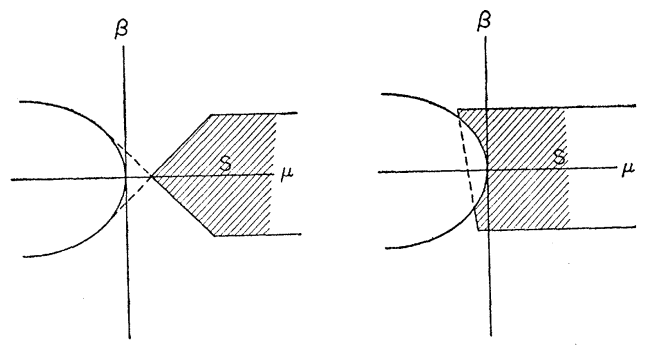

Fig. 2. The region $\delta$, the support of the weight function $H(\mu, \beta)$, in the $(\mu, \beta)$ plane.

Green's function, since in the physical region the contributions from the two orderings of the commutator are quite distinct. Consequently the representation of the Fourier transform of the retarded commutator is identical to that for the time-ordered Green's function provided one drops the sign factor associated with the infinitesimal imaginary term. One obtains

$$
G_{+}(k)=\int_{\mathcal{S}} d \beta d \mu \frac{H(\mu, \beta)}{k^{2}+2 p k \beta-\mu+i \epsilon}
$$

(for case $A$ only).

We cannot write the time-ordered Green's function for case $B$, however. In this case the two physical regions in the $\left(k^{2}, 2 p k\right)$ plane cross. The mass condition for this to happen is $2 \kappa \geqslant M_{1}+M_{2}$. Here, although the single particle $\kappa$ may be stable, the assertion of nonvanishing matrix elements connecting the particle to the states $M_{1}$ and $M_{2}$ is the assertion that the quantum numbers of the particles are such that two $\kappa$ particles may spontaneously interact to form the combination of states $M_{1}$ and $M_{2}$. The commutator, and thus the causal Green's function, does not contain all the information, since we cannot isolate from this commutator the time-ordered product of the operators. This is in close analogy to the scattering from excited or unstable systems, in which the causal response does not determine the free decay.

\section{FURTHER SPECTRAL. INFORMATION : THE "BOUND STATE" TERM}

There is still another item of information in the spectral decomposition that we can explicitly impose upon our representation. If the state of least mass occurring in an ordering of the commutator is a singleparticle state isolated by a finite gap from the states of higher mass, which often is the case if we ignore the electromagnetic interactions, we know that the Fourier transform of the commutator, $f\left(k^{2}, p k\right)$, will vanish in the physical region for all values of the invariants $k^{2}$ and $p k$ that lie between the single line in the $\left(k^{2}, 2 p k\right)$ plane associated with the single-particle state and the beginning of the continuum states. This additional information cannot be simply expressed as the vanishing of the weight function in a region larger than the one 
we have found. Our arguments lead us to the smallest convex support for $H(\mu, \beta)$ but no further. As we shall see, this new information takes the form of an integrability condition on the weight function and allows us to integrate away the weight function in the region of the $(\mu, \beta)$ plane associated with this new region in the $\left(k^{2}, 2 p k\right)$ plane at the expense, in the Green's function, of a new denominator. To exploit this structure we shall modify this term, write a new representation for the modified functions, and then reinstate this contribution.

As an example of such a structure, let us suppose that, in the first ordering of the commutator, the mass $M$ is a single-particle mass and the continuum begins with a mass $M_{1}>M$. The term in the commutator arising directly from the single-particle state is

$$
\begin{aligned}
f\left(k^{2}, p k\right)=2 \pi \theta\left(p^{2}+p k\right) \delta( & \left.(p+k)^{2}-M^{2}\right) \\
& \times \sum_{q}\left\langle p\left|j_{1}\right| q\right\rangle\left\langle q\left|j_{2}\right| p\right\rangle+\cdots,
\end{aligned}
$$

where $q=k+p$, and the sum is over the other quantum numbers of the single-particle state. The other term that makes up the expected $\epsilon\left(p^{2}+p k\right)$ structure arises in the other order of the commutator from states containing three particles, one of mass $M$ and a particle and antiparticle of mass $\kappa=\left(p^{2}\right)^{\frac{1}{2}}$. This $\delta$-function term may be removed by defining a new function:

$$
\bar{f}\left(k^{2}, p k\right)=\left[(k+p)^{2}-M^{2}\right] f\left(k^{2}, p k\right) .
$$

This $\bar{f}$ function will now vanish everywhere in the physical region except in the regions defined by the thresholds of the continua $M_{1}$ and $M_{2}$. The modified function in coordinate space:

$$
\bar{C}(x)=\left\{-\square^{2}+2 i p_{\mu} \partial^{\mu}+p^{2}-M^{2}\right\} C(x)
$$

is still a causal function. We write a new representation for $\bar{f}$ :

$$
\begin{aligned}
f\left(k^{2}, p k\right)=\int_{\mathcal{S}^{\prime}} & d \beta d \mu \\
& \quad \times \epsilon\left(p k+\beta p^{2}\right) \delta\left(k^{2}+2 p k \beta-\mu\right) \bar{H}(\mu, \beta)
\end{aligned}
$$

with the limits

$$
\delta^{\prime}: \mu \geqslant \lambda\left(M_{1}, M_{2}, \beta\right) ; \quad 1 \geqslant \beta \geqslant-1 .
$$

We turn now to the retarded Green's function and consider the action of our polynomial factor upon it.

$$
\begin{aligned}
& \left\{(k+p)^{2}-M^{2}\right\} G\left(k^{2}, p k\right)=-i \int d x \theta(x) \bar{C}(x) e^{i k x} \\
& +\int d x e^{i k x} \delta\left(x_{0}\right)\left(2 p_{0}+k_{0}+i \partial_{0}\right) C(x) .
\end{aligned}
$$

The first term is the retarded Green's function associated with the modified commutator. The second term arises from the boundary during the integration by parts and is, by invariance, causality, and locality, at worst a polynomial in $k^{2}$ and $p k$ whose structure can be determined from the canonical commutation relations and the field equations. We set

$$
\int d x e^{i k x} \delta\left(x_{0}\right)\left(2 p_{0}+k_{0}+i \partial_{0}\right) C(x)=P\left(k^{2}, p k\right) .
$$

Then evaluation of the retarded integral in (16) leads to

$$
\begin{aligned}
& \left\{k^{2}+2 p k+p^{2}-M^{2}\right\} G\left(k^{2}, p k\right) \\
& \quad=\int_{\mathcal{S}^{\prime}} d \beta d \mu \frac{\bar{H}(\mu, \beta)}{k^{2}+2 p k \beta-\mu+i\left(p k+\beta p^{2}\right) \epsilon}+P\left(k^{2}, p k\right) .
\end{aligned}
$$

Since the right-hand side of (16) is analytic in the upper half $k_{0}$ plane for all physical momenta, the lefthand side is also analytic above the real $k_{0}$ axis and we deduce:

$$
\begin{aligned}
G\left(k^{2}, p k\right)= & \frac{P\left(k^{2}, p k\right)}{\left\{(k+p)^{2}-M^{2}\right\}+i\left(p^{2}+p k\right) \epsilon} \\
& +\frac{1}{\left\{(k+p)^{2}-M^{2}\right\}+i\left(p^{2}+p k\right) \epsilon} \\
& \times \int_{\Phi^{\prime}} d \beta d \mu \frac{\bar{H}(\mu, \beta)}{k^{2}+2 p k \beta-\mu+i\left(p k+\beta p^{2}\right) \epsilon}
\end{aligned}
$$

This representation makes explicit all of the information we can deduce from the mass spectrum and embodies the tightest restrictions on the range of $\mu$ and $\beta$ that we have been able to draw. If the two denominators were combined, this representation could be reduced to the form (1) with a concomitant increase in the ranges of the parameters in the weight function.

A way of re-isolating the "bound-state" term is to extract from (19) the imaginary part, that is, the commutator:

$$
\begin{gathered}
C\left(k^{2}, p k\right)=\epsilon\left(p^{2}+p k\right) \delta\left((k+p)^{2}-M^{2}\right)\left\{\Lambda\left(k^{2}\right)\right. \\
\left.+P\left(k^{2}, \frac{1}{2}\left(M^{2}-p^{2}-k^{2}\right)\right)\right\}+\rho \frac{1}{(k+p)^{2}-M^{2}} \\
\quad \times \int_{\mathcal{S}^{\prime}} \epsilon\left(p k+\beta p^{2}\right) \delta\left(k^{2}+2 p k \beta-\mu\right) \bar{H}(\mu, \beta) d \beta d \mu .
\end{gathered}
$$

Here

$$
\begin{aligned}
\Lambda\left(k^{2}\right) & =\odot \int_{\mathcal{S}^{\prime}} d \beta d \mu \frac{\bar{H}(\mu, \beta)}{k^{2}(1-\beta)-\mu+\beta\left(M^{2}-p^{2}\right)} \\
& =\odot \int_{\sigma_{0}}^{\infty} \frac{\mathcal{H}(\sigma) d \sigma}{k^{2}-\sigma},
\end{aligned}
$$

where

$$
\mathscr{C}(\sigma)=\int d \beta d \mu \frac{\bar{H}(\mu, \beta)}{1-\beta} \delta\left(\sigma-\frac{\mu-\left(M^{2}-p^{2}\right) \beta}{1-\beta}\right),
$$


and

$$
\begin{aligned}
\sigma_{0}= & \min \{\sigma: \mu, \beta \text { in } \delta\} \\
= & \frac{\left(M^{2}-\kappa^{2}\right)\left(M_{1}-M_{2}\right)+2 \kappa\left(M_{1}-\kappa\right)\left(M_{2}-\kappa\right)}{M_{1}-M_{2}+2 \kappa} \\
& \text { for } M_{1}>M>2 \kappa-M_{2} \\
= & \frac{1}{2}\left(M_{1}^{2}+M_{2}^{2}\right)-\kappa^{2} \text { for } 2 \kappa-M_{2}>M .
\end{aligned}
$$

The value of $\sigma_{0}$ represents two things. It is the point at which, as $k^{2}$ increases from spacelike values, $\Lambda\left(k^{2}\right)$ ceases to be an analytic function of $k^{2}$, and it is the point along the line $k^{2}+2 p k+p^{2}=M^{2}$ at which the second $\delta$ function in (20) begins to be satisfied. In the case corresponding to (21.1), $\sigma_{0}$ lies in the nonphysical region of the $\left(k^{2}, p k\right)$ plane, in (21.2) it lies in the physical region. In the physical region, for values of the invariants that satisfy only the first $\delta$-function in (20) and not the second, $\Lambda\left(k^{2}\right)+P\left[k^{2},\left(M^{2}-k^{2}-p^{2}\right) / 2\right]$ is the product of matrix elements corresponding to the product of two vertices in (12). Thus we have shown that this product is real and analytic as a function of $k^{2}$ for $k^{2}<\sigma_{0}$. Using the representation of $\mathrm{I}$ for the vertex we could show that this product of vertices was, in general, analytic beyond $\sigma_{0}$ up to some $\sigma_{1}$. Therefore, between $\sigma_{0}$ and $\sigma_{1}$, the weight function $\mathcal{H C}(\sigma)$ in the representation for $\Lambda\left(k^{2}\right)$ vanishes, since the latter is defined everywhere as a principal part integral.

Since we shall, in the next section, explore the analytic properties of this representation for the retarded Green's function as a function of $p k$, we rewrite the representation by adding and subtracting from the integrand the term $\odot[\bar{H}(\mu, \beta)] /\left[k^{2}(1-\beta)+\left(M^{2}-p^{2}\right) \beta\right.$ $-\mu]$ to obtain

$$
\begin{gathered}
G\left(k^{2}, p k\right)=\frac{\Lambda\left(k^{2}\right)+P\left(k^{2}, p k\right)}{\left[(k+p)^{2}-M^{2}\right]+i\left(p^{2}+p k\right) \epsilon} \\
-\beta \int_{\mathcal{S}^{\prime}} d \beta d \mu \frac{\beta \bar{H}(\mu, \beta)}{\begin{array}{l}
\left\{\left(k^{2}+2 p k \beta-\mu\right)+i\left(p k+\beta p^{2}\right) \epsilon\right\} \\
\times\left\{k^{2}(1-\beta)+\left(M^{2}-p^{2}\right) \beta-\mu\right\}
\end{array}} .
\end{gathered}
$$

\section{FORWARD DISPERSION RELATIONS}

Simple applications of our integral representations are the dispersion relations for the forward scattering amplitude. ${ }^{5}$ The physical values of this amplitude are obtained directly from the representation by putting the projectile momentum on the mass shell, taking $k^{2}=m^{2}$. As an example of the behavior of the bound state term, consider (22). The first term in (22) then has the form of a simple pole in the variable $p k$, the pole occurring in general outside the physical region of $p k$ : $(p k)^{2} \geqslant p^{2} m^{2}$. The residue of this pole is simply related, as we have seen, to the analytic continuation

\footnotetext{
${ }^{5}$ M. L. Goldberger, Phys. Rev. 97, 508 (1955).
}

of the vertex function and can be used in the usual way to define the coupling constant.

The rest of the $T$-matrix function has the form

$$
\int_{\mathcal{S}^{\prime}} d \beta d \mu \frac{H(\mu, \beta)}{\left(m^{2}+2 p k \beta-\mu\right)+i\left(p k+\beta p^{2}\right) \epsilon}
$$

where $\mathcal{S}^{\prime}$ is given by (15).

This has the form

$$
\begin{gathered}
\int d \sigma\left\{\frac{h_{1}(\sigma)}{p k-\sigma+i \epsilon}+\frac{h_{2}(\sigma)}{p k-\sigma-i \epsilon}\right\} \\
h_{1}(\sigma)=\int_{\mathcal{S}^{\prime}} d \beta d \mu \frac{\bar{H}(\mu, \beta)}{2 \beta} \delta\left(\sigma-\frac{\mu-m^{2}}{2 \beta}\right) \theta\left(\frac{p k}{\beta}+p^{2}\right), \\
h_{2}(\sigma)=\int_{\mathcal{S}^{\prime}} d \beta d \mu \frac{\bar{H}(\mu, \beta)}{2 \beta} \delta\left(\sigma-\frac{\mu-m^{2}}{2 \beta}\right) \\
\times \theta\left(-\frac{p k}{\beta}-p^{2}\right) .
\end{gathered}
$$

If $m^{2} \geqslant \max \left\{\left(M_{1}-\kappa\right)^{2},\left(M_{2}-\kappa\right)^{2}\right\}$, then both $h_{1}$ and $h_{2}$ are in general not zero and no analytic information can be deduced. In the special case of symmetrical masses (pion-nucleon scattering), if $m^{2}=\left(M_{1}-\kappa\right)^{2}=\left(M_{2}-\kappa\right)^{2}$, then $h_{2}(\sigma) \equiv 0$ and $h_{1}(\sigma)$ vanishes if $|\sigma|<\kappa m$. This is then directly the form embodied in the dispersion relation and we can deduce that $T(p k)$ is analytic in the upper half plane.

The other important special case arises when the particle can react exothermically in one order. Then $m^{2}=\left(M_{1}-\kappa\right)^{2} \geqslant\left(M_{2}-\kappa\right)^{2}$ and we have the following structure:

$$
\begin{array}{r}
T(p k)=\int_{\kappa m}^{\infty} d \sigma \frac{h_{1}(\sigma)}{p k-\sigma+i \epsilon}+\int_{\kappa\left(M_{1}-\kappa\right)}^{\kappa m} d \sigma \frac{h_{1}(\sigma)}{p k-\sigma+i \epsilon} \\
+\int_{0}^{\kappa\left(M_{1}-\kappa\right)} d \sigma\left\{\frac{h_{1}(\sigma)}{p k-\sigma+i \epsilon}+\frac{h_{2}(\sigma)}{p k-\sigma-i \epsilon}\right\} \\
+\int_{-\infty}^{0} d \sigma \frac{h_{1}(\sigma)}{p k-\sigma+i \epsilon} .
\end{array}
$$

Although the function $T(p k)$ is not an analytic function of $p k$, it is only in the nonphysical region that the cut lines lie on both sides of the real axis in the $p k$ plane. For physical values of $p k$, the denominator of the term in $h_{2}$ never vanishes. Changing the sign of $i \epsilon$ in this term defines a new function, $\widetilde{T}(p k)$, analytic in $p k$ in the upper half plane. This representation is again directly a dispersion relation, in this case for nucleon-nucleon scattering and $K$-nucleon scattering. These relations differ from those that have been suggested ${ }^{6}$ in that the

${ }^{6}$ Goldberger, Oehme, and Nambu, Ann. Phys. 2, 226 (1957); P. 'T. Matthews and A. Salam, Phys. Rev. 110, 565 (1958); C. J. Goebel, Phys. Rev. 110, 572 (1958); K. Igi, Progr. Theoret. Phys. (Kyoto) 19, 238 (1958). 
weight function does not vanish in part of the nonphysical region.

Other cases can easily be derived from the form of $\delta$. Dispersion relations for the spin-flip amplitude ${ }^{7}$ or for the derivatives of the amplitude with respect to the angle evaluated in the forward direction may be obtained by using representations of this type to exhibit their structure as Fourier transforms of causal functions with specified mass spectra.

\section{CONCLUDING REMARKS}

In the previous sections we have shown how the information contained in the mass spectrum can be used to construct an integral representation with real weight functions for diagonal matrix elements of various products of two field operators. We have throughout worked with manifestly covariant expressions, and this applies in particular to the method of imposition of the lowest mass limits. The symmetric point-to-line correspondence between the $\left(k^{2}, p k\right)$ plane and the $(\mu, \beta)$ plane affords a rapid graphical method of defining the support of the weight function $H(\mu, \beta)$, carried through in Sec. III. The weight function as obtained is unique and no further support conditions can be imposed on it from our axioms alone. The representation does not necessarily exhaust the contents of these axioms.

One has the freedom of redefining the continuation to the unphysical region of any physical function, and, depending upon the specific application, the continuation could be taken differently. For any continuation other than that here used, however, a representation of the form of Eq. (1) would require an unbounded range

\footnotetext{
${ }^{7}$ A. Salam and W. Gilbert, Nuovo cimento 3, 607 (1956);
} R. Oehme, Phys. Rev. 100, 1503 (1955). in $\beta$; in that case, our method of incorporating the máss restrictions as support conditions on the weight function could not be carried through. We have exploited the freedom in the continuation to the unphysical region in the construction of the forward dispersion relations by "moving" the cut lines in the unphysical region to effect a trivial analytic continuation. This method enables us to construct dispersion relations for nucleon-nucleon and $K$-meson-nucleon scattering. Unlike the situation in the dispersion relations which have been conjectured before ${ }^{6}$ the analytic scattering amplitudes in our relations are nonvanishing throughout the unphysical region $-\kappa m<p k<\kappa m$. For the amplitude to vanish in certain parts of the unphysical region, additional specification seems necessary. As far as the general theory is concerned, symmetric mass restrictions (say, pion scattering) are, in this sense, essentially distinct from unsymmetric mass restrictions (say, nucleon-nucleon scattering).

It is to be noticed that, after specialization of the projectile to the mass shell, one should expect the structure of the amplitude to be symmetric between the two particles; and this is in fact true of both the specialized function $T(p k)$ and the modified function $\tilde{T}(p k)$.

If the state $|p\rangle$ is a one-particle state, further analytic information may be obtained: the possibility of associating a local field with a one-particle state $|p\rangle$ implies information on the dependence of the matrix element, and consequently of the weight function, on $p^{2}$. Similarly, the fact that, after separation of the bound states, the contributing intermediate states contain two or more particles may impose further restrictions on $H(\mu, \beta)$. These matters will be treated elsewhere. 